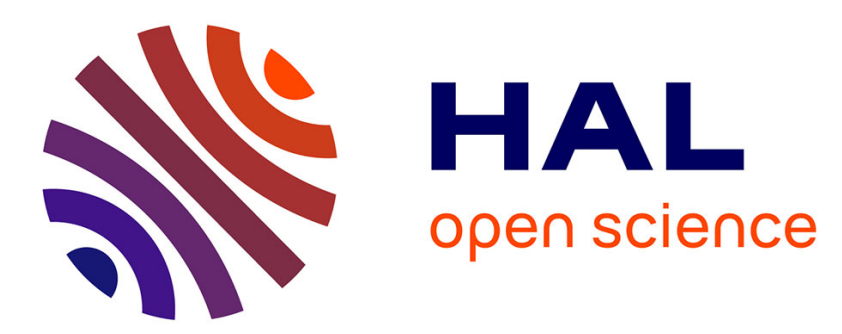

\title{
Introduction to personalisation in cyber-physical-social systems
}

\author{
Bereket Abera Yilma, Yannick Naudet, Hervé Panetto
}

\section{To cite this version:}

Bereket Abera Yilma, Yannick Naudet, Hervé Panetto. Introduction to personalisation in cyberphysical-social systems. OTM/IFAC/IFIP International Workshop on Enterprise Integration, Interoperability and Networking, EI2N 2018, DOA Institute, Oct 2018, La Vallette, Malta. pp.25-35, 10.1007/978-3-030-11683-5_3. hal-02009913

\section{HAL Id: hal-02009913 \\ https://hal.science/hal-02009913}

Submitted on 6 Feb 2019

HAL is a multi-disciplinary open access archive for the deposit and dissemination of scientific research documents, whether they are published or not. The documents may come from teaching and research institutions in France or abroad, or from public or private research centers.
L'archive ouverte pluridisciplinaire HAL, est destinée au dépôt et à la diffusion de documents scientifiques de niveau recherche, publiés ou non, émanant des établissements d'enseignement et de recherche français ou étrangers, des laboratoires publics ou privés. 


\title{
Introduction to Personalisation in Cyber-Physical-Social Systems
}

\author{
Bereket Abera Yilma ${ }^{1,2}$, Yannick Naudet ${ }^{1}$, and Hervé Panetto ${ }^{2}$ \\ 1 Luxembourg Institute of Science and Technology (LIST), Luxembourg \\ ${ }^{2}$ Université de Lorraine, CNRS, CRAN, France
}

\begin{abstract}
In this paper we introduce the notion of personalisation in Cyber-Physical-Social Systems (CPSS). A CPSS is an extension of Cyber-Physical systems involving cyberspace and physical space, in which humans, machines and objects interact adding to the complexity of the system, especially due to the dynamics of human behaviour that is not yet fully understood. We propose Personalisation to address this complexity. Since the development of CPSS is still at its infancy, we first cover a brief overview on the existing conceptualizations of CPSS and present the perspective we take for our work. Then we discuss the benefits of introducing Personalisation in CPSS and formalize the notion. The discussion further illustrates research challenges for Personalisation in CPSS with examples on possible use cases, taken from preceding works as well as future ones.
\end{abstract}

Keywords: Personalisation - Recommender System - Cyber-Physical Social-Systems · Cobotics.

\section{Introduction}

From a general perspective, CPSS is a system comprising three intertwining subsystems: the Cyber, the Physical and the Social systems. The European Commission contributed a major part for the emergence of the Internet of Things (IoT) paradigm from a computer science perspective, which played an indispensable role for the orchestration of the physical and cyber systems with the goal of making tools and services intelligent by connecting tools and electronic equipments to the Internet so as to develop a network of computers and objects that are capable of self configuration [20]. On the other hand the US National Science Foundation (NSF) initially supported the notion of CPS derived from an engineering perspective, with the objective of controlling and monitoring physical environments and phenomena via the integration of sensing, computing, and actuating devices [21]. Despite their initial philosophical difference, IoT and CPS share many similarities hence they have been used interchangeably without a clearly defined demarcation. There is however a fundamental difference that should be highlighted: the fact that a CPS refers to a particular system explicitly, while IoT refers at the same time to the concept, the system formed by all the connected devices and a particular system of interconnected objects. As a system, a CPS 
typically collects and controls information about phenomena from the physical world through networks of interconnected devices, in order to achieve its objective. Originally, humans are assumed as external entities interacting with the system. Over the years the increasing use of smart phones and the tight link with their users has lead CPS systems to exploit the large number of such users as a multifaceted source of information, i.e. human sensors. Subsequent research studies have then recognized the importance and perspectives open by a new, Human-in-the-Loop (HitL) CPS paradigm [2], where humans are intrinsic actors of the system. Different techniques have then been used to introduce human actors in CPS, paving the way for the foundation of Cyber-Physical-Social Systems(CPSS). The development of CPSS, where social refers to the human aspects, is still in its infancy, especially because it often faces inevitable system instabilities, mainly due to the fact that human's actions and behaviour are the result of individual preferences, cognition, motivation and other natural or/and environmental factors. Moreover, each person is unique and might not follow system's rules that are not aligned with his way of thinking, convictions, etc. Indeed, the human behaviour is driven by complex phenomena that we do not fully understand and are still difficult to predict and manage compared to machines and softwares which are made by humans and usually prone to errors. To ensure a good functioning of CPSS, means are needed, to some extent, to have more control on the human system, while keeping individuals' freedom to operate. We think that the personalisation might be a solution; personalisation of IT services and devices, or more generally of Human-Machine Interaction. Our postulate is that personalisation provides a way to have more control over CPSS in such a way human behaviour does not interfere with the system's functioning, that is optimized for individual's satisfaction by adapting to individual's characteristics, needs, capabilities and preferences. There are several opportunities where the CPSS would benefit from, both for a better functioning of the system (the CPSS) and the user experience. The purpose of this paper is to make a first step in introducing the notion of personalisation in Cyber-Physical-Social Systems by discussing its added values, capabilities, applications, and by examining the associated issues and scientific challenges. The rest of this paper is organized as follows. Section 2 covers a brief literature review on CPSS, highlighting the common views and the widely accepted current conceptualizations of a CPSS. Section 3 presents the concept of personalisation in CPSS, and the problems and opportunities that personalisation brings when applied to CPSS. Section 4 presents illustrative Use-cases, their associated issues and scientific challenges for personalisation in CPSS. Section 5 proposes a concluding discussion.

\section{Cyber-Physical-Social Systems}

CPSS encompass Cyber, Physical and Social systems. Recent foundational works [6, 7] presented it as an evolution of the preexisting notion of Cyber-Physical system (CPS) where Socio-technical aspects are added. In the following section we 
present a brief overview of the main conceptualizations of CPSS that appear in the literature.

\section{$2.1 \quad$ State-of-the-art}

In this section we present a brief literature review on CPSS, organized in two main categories. In the first category we introduce the different ways researchers define CPSS, whereas in the second we present the two main views conceptualizing the social aspect in CPSS.

A common understanding, shared by most of the works in the literature or constituting at least a common ground, is that CPSS is a system comprising three intertwining subsystems (i) The human-based system which refers to the social system containing human actors and their interconnected devices/agents and/or social platforms providing human-based services, (ii) The software-based system that refers to the cyber world providing software-based services including the underlying infrastructures and platforms, either on-premise or in the Cloud and (iii) The thing-based systems referring to the physical world that includes sensors, actuators, gateways and the underlying infrastructures [1,3, 7, 9-13].

Different definitions have also been proposed. Particularly, in [4] CPSS has been defined as the integration of CPS and CSS (Cyber-Social System) to enable smart interaction between cyber, physical and social spaces, where CPS includes communicators, multimedia entertainment and business processing devices, etc. and CSS refers to social networks such as Facebook, Twitter, Youtube, etc. In [4] CPSS has also been described as the extension of IoT/CPS in the presence of humans interacting with CPS and other users.

In [8] and [19], the following definition is used: "CPSS is a system that captures a synergetic interaction between computing and human experience while providing holistic computational solutions encompassing the PCS (Physical, Cyber and Social) dimensions." Some works have tried to analyze the social aspect further by subdividing it further into Social space and thinking space. [10] introduces the acronym CPST (Cyber-Physical-Social-Thinking hyperspace), a system established through the emergence of the new dimension of thinking space in the CPS space. The thinking space is related to high-level thoughts or ideas raised during intellectual activities.

The way of defining CPSS is clearly use-case dependent and not homogeneous. However, despite the existence of various definitions, all agree on the presence of humans as an integral part of the system. Depending on the meaning they associate to the social aspect of CPSS, most works adopt one of the two views discussed below.

1. Human as a sensor: $[5-7,13]$ conceptualize the social aspect by considering humans as information sources, i.e. sensors. This view of conceptualization primarily focuses on fusing various information originating from the social space (humans and their observations) with cyber-systems and physicalsystems in order to accommodate various application needs. 
2. Human as a system part: [1-3, 8, 12] On the other hand most of the recent works intend to conceptualize the social aspect of CPSS not only by considering humans as social sensors but also as co-creators being an integral part of the system. It is also known as the human-centric way of conceptualizing CPSS [8]. This way of conceptualization considers humans as full members of the CPSS, involving observations, experiences, background knowledge, society, culture and perceptions (i.e human intelligence and social organizations (e.g. Communities)) in order to co-create products and services together with the CPS. Here, humans play the role of resources in that they provide information, knowledge, services, etc., which at the same time they consume, thus becoming users of the CPSS. Hence, the human aspect is at the center of the system design, to provide user centered services.

\subsection{Definitions}

Following the state of the art analysis, we propose to adopt a definition and conceptualization for CPSS that is grounded on the generic framework provided by the theory of systems. From this systemic perspective, a CPSS can be understood as an environment, or system, where humans and machines evolve in both the physical and the virtual world, interacting all together. As illustrated by Figure 1, the elements of a CPSS are its three interconnected systems, the Cyber, the Physical and the Social, which we represent as CPS and Social System, which interact in an environment composed by the physical and the virtual spaces. In an effort to a generic and uniform understanding of CPSS, we propose the following three definitions.

Definition 1: CPSS. A system strictly composed of a CPS, a Social System, a virtual space and a physical space, in which the systems components interact, where CPS and Social System are defined respectively as follows.

Definition 2: CPS. A system encompassing all the systems and subsystems of Cyber and Physical Systems, their components and the interaction between them, as well as integrations of computation with physical processes $[2,4,6,10]$.

Definition 3: Social System. A system that comprises interacting individuals, having each their own cognition, preferences, motivation and behaviour.

In the following subsection we briefly cover Crowd systems which to a certain extent can be considered as a CPSS.

\subsection{CPSS and Crowd Systems}

Crowd systems are systems where a large group of people interact with an information system either in virtual or physical spaces towards the fulfillment of the system's objective [16]. As it is the case in CPSS, the complexity of crowd systems arises from the existence of a human factor which can never be fully controlled. As a result, certain inconsistencies and systemic instabilities always remain in crowd systems. Examples of such systems are crowd-sourcing platforms, but also Wikis, or museums equipped with smart guidance systems. As a CPSS some crowd systems also involve CPS and Human systems interacting in 


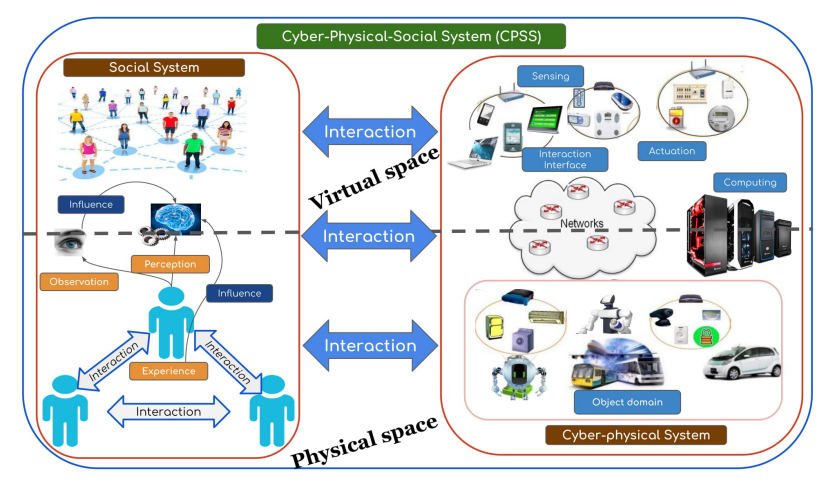

Fig. 1. Elements of a Cyber-Physical and Social System

Virtual and physical spaces, therefore to a certain extent, crowd systems can be considered as CPSS. However due to the fact that the interaction do not always involve physical spaces, not all crowd systems can be considered as a CPSS. Nevertheless, personalisation has been recently proposed in [16] as a tool to mitigate the inconsistencies arising from the human involvement in crowd systems, so as to improve system performance and provide better services. We build on this proposal to extend this idea to the domain of CPSS, since similar challenges are shared, but in a broader context involving physical spaces.

\section{Personalisation in CPSS}

Personalisation, broadly known as customization, refers to tailoring a service or a product in a way that it fits to specific individuals' preferences, cognition, needs or capabilities under a given context. In some cases, it also targets a group of people sharing a common context. Strategies of personalisation primarily depend on the a-priori knowledge and understanding of the target individual or group so as to build a user model and latter apply the related customization. We strongly believe that it can be used in CPSS to mitigate challenges imposed by the human behaviour, cognition and preferences. In the following, we provide a high-level formalization of personalisation in CPSS, defined as an optimization problem.

\subsection{Problem formalization}

In general inconsistencies imposed by user's behaviour and preferences, together with constraining factors and other co-existing possibly divergent systemic objectives make the CPSS rather complex. Therefore, efficient means are needed to keep the CPSS equilibrium by fulfilling objectives respecting its constraints while at the same time fulfilling the objectives and interests of humans inside. Personalisation in CPSS needs to take into account all the constraints from the environment but the main objectives are those of the user (e.g. matching interests or preferences) and of the application (i.e. personalisation provider). This 
particular scenario can be formalized as a constrained multi-objective optimization problem. Providing that a personalized service generally implies making the best possible trade-offs between user interests and the general objectives. A classical personalisation problem can be formalized [22] as a function of a user $u$, the IT application $a$ which implements the specific personalisation objective pursued and a context $c$ (a set external elements) related to $u$ or $a$ which can have an influence on the personalisation objective (e.g. rules regarding the kinds of users, regulations, etc.), written as $P_{u}^{(\text {classic })}=f(u, a, c)$. In a CPSS, there exist multiple coexisting objectives, influencing each other and constrained by different factors from the external environment and adding to the complexity of the personalisation problem. The user evolves in a physical space, with other persons. His behaviour is constrained by the first, while influencing and being influenced by the later. Additionally, the physical space has itself a given purpose, calling for expected specific behaviours of people inside. This particular context leads to formulate the personalisation problem differently, as a function of the user $u$, the physical space $s$, the crowd of other persons in the physical space $\mathrm{cr}$, the IT application implementing personalized services $a$ and the global context $c$, the latter being feed typically by sensors' observations written as:

$$
P_{u}^{(C P S S)}=f(u, s, c r, a, c)
$$

To illustrate this function, we can take the case of Personalized recommendations, which is a particular case of personalisation. In CPSS, with equation (1) rewritten as $P R_{u}^{(C P S S)}=f(u, I, s, c r, a, c)$, it can be defined as the problem of finding the subset $I r e c_{u} \subset I$ that an application $a$ can suggest to a user $u$, under the set of constraints $C O=C O_{u} \cup C O_{s} \cup C O_{c r} \cup C O_{c}$ induced by $u$, the space $s$ in which he evolves, the crowd $c r$ to which he belongs and the influencing context $c$, and fulfilling at best the set of objectives $O=O_{u} \cup O_{s} \cup O_{a}$ linked to $u, s$ and $a$.

\section{Illustrative Use-Cases}

In the following sections we illustrate the notion of personalisation in CPSS through different use cases with the goal of opening a new perspective for building personalized IT services in future physical spaces designed as CPSS.

\subsection{Personalized guidance in exhibition areas}

Exhibition areas are normally composed of a large number of items to be visited commonly refereed as points of interest (POI). They exist in the physical space, containing visitors having different visiting style and preferences and carrying their sensor-enabled smart devices, all together in one environment: a $\boldsymbol{C P S S}$. In such sites visitors usually miscalculate their available time and spend wondering around exhibition sites, sometimes following different recommendations biased by many sources without visiting items that would have been interesting to them. 
Introducing personalized services in such environments guiding visitors to their presumed POIs delivers a great deal of benefits to both the exhibition center and the visitors. However doing so is far from trivial not only because of the inconsistencies imposed by humans behaviour and preferences but also the existence of the IT services and the visitors together in a physical environment by itself brings additional constraints such as congestion around popular exhibits, the size of the museum and the number of items to be visited which is normally subject to available time of visitors. Apart from exhibition center's general objective of reducing congestion and increasing visitor satisfaction there might also be an objective of making less popular items to be visible. Another important scenario in exhibition areas is the case of personalized recommendation for a group visit, where each group comprises individuals having their own interests, but behaving as a single entity, the group. This is the typical scenario of the Social Choice problem, that a group of people arrive at a saddle point where a group-wise recommendation has to be provided that satisfies some notion of consensus among the group. Incorporating all these aspects in making a personalized guidance is a complex problem which has not yet been fully addressed. Some experimental results from preceding works indicated that personalized path recommendations increased the perceived visitor satisfaction and reduced congestion $[15,16]$ in ex-

hibition areas like Museums. Nevertheless personalisation in physical spaces is rare due to the existence of multiple objectives and numerous constraining factors. We believe the formulation of the problem as a constrained multi-objective optimization could open a new perspective and bring us a step forward in introducing personalisation to the domain of exhibition areas for providing a better visitor experience.

\subsection{Personalisation in Cobotics}

Industry 4.0 , often refereed to as the $4^{\text {th }}$ industrial revolution is a terminology for the emerging advent in manufacturing and automation technologies which includes CPS and humans interacting in a physical environment which could be seen as a typical CPSS. Our interest in this particular domain is the increasing trend of using Cobots in such manufacturing and automation facilities. The term cobotics is formed by joining the terms collaborative and robotics to imply the direct interaction between humans and robots [17]. A Cobot is defined as a robot that has been designed and built to collaborate with humans in various application domains such as industrial, domestic, medical, military, etc. for performing specific tasks such as, moving and carrying objects, transporting, assembling, surface processing, welding, cutting engraving, etc. [18]. Even though the Cobots are designed to perform a desired task, their duty in collaborating with humans might not be a satisfying experience to the human users. Despite the collaborating humans are assumed to possess similar set of skills and qualification towards the desired goal, their individual preference, cognition, motivation and other natural factors could result in an unenjoyable collaboration experience. This could lead to reduced system performance and worst case scenario failure to achieve the desired objective. In this particular scenario the main source of systemic 
instability comes from the complexity imposed by human nature. In addition the various industrial objectives and their constraining factors add up to this complexity. We believe introducing personalisation in this domain could greatly benefit the overall system performance and also user experience by customizing the system according to one's preference, cognition and behaviours.

\subsection{Issues and Challenges of personalisation in CPSS}

Personalisation in CPSS is subject to a number of challenges arising from the four components of CPSS in addition to the classical issues like, e.g. cold start, user profiling and situation identification. Since it is a broad subject which covers and tries to bring together concepts from different research areas mainly personalisation, Social systems, Cognitive science, Recommender systems, CPSS and $C P S$, it is vulnerable to different issues and challenges originating from these fields. The orchestration of various concepts from different fields by itself constitute a challenge adding to the complexity of the problem. In the following, we highlight some of these issues and challenges specific to Personalisation in CPSS.

Interoperability Challenges: As the personalisation in CPSS is a broad notion encompassing various systems, subsystems, their components and different devices, efficient interoperability is an indispensable requirement for satisfying the overall Personalisation objectives. Indeed, different challenges arise with the orchestration of these components. For instance communication related challenges, tool selection challenges and data related challenges are among the main ones. Personalized recommendations mainly relies on data gathering about the users of personalized services, which is collected through different sensing sources (devices). Furthermore the question of which tools or sensing sources to employ, which data features to use and which techniques (algorithms) to adopt in different use cases is left for the personalisation engineer to decide. This as clearly an impact on the personalisation quality. Hence at the heart of Personalisation in CPSS, smart and context-aware interoperability is a prerequisite.

Privacy and compliance Challenges: Providing personalized services in CPSS essentially requires the acquisition of Personal data of users. However it is compulsory to follow privacy rules by being completely transparent. There are a number of regulatory restrictions on tracking user's behaviour and accessing personal data of users. In this regards different countries have different rules. Users of personalized services expect service providers to know their preferences and needs. Therefore introducing personalisation in CPSS also faces a challenge to find a proper balance between the satisfaction of users with personalized offerings and organizational objectives, while keeping data secure. The data protection regulations are an important component of the context in a personalisation problem (the generic context $c$ in equation (1)), which generate constraints and objectives that need to be taken into account.

Human complexity Challenges: Human behaviour is a complex piece of the puzzle that is not yet fully understood. Providing personalized offerings to individuals, as such, impacts the CPSS where the individuals evolve. This in 
turn drives or limits their actions. Additionally human's actions and behaviour are demonstrations induced by individual preferences, cognition, motivation and other natural or/and environmental factors. On top of this, social choice scenarios like the personalized group tour recommendation, where a group comprises individuals having their own interests, but behaving as a single entity requires making group-wise decision that satisfies some notion of consensus among the group through deep and complex analysis of different preferences. Personalisation requires the modelling of human behaviour, accounting for the multiple kinds of interactions people have with the components of a CPSS. This is the main prerequisite to be able to use personalisation as a tool for building more efficient, more stable or more smart CPSS.

\section{Conclusion}

In this introductory paper we proposed an approach to introduce the notion of personalisation in CPSS which opens an interesting perspectives for the evolution and improvement of CPSS. Our approach primarily intends to provide a way for controlling the complexities caused by human behaviour in CPSS. We formalized the notion as solving a constrained multi objective optimization problem for the future to work on modeling and experimentally analyzing use cases involving physical spaces designed as CPSS. The perspective contributes both to the fields of CPSS, and personalisation/User Modelling/Recommender Systems where application to the physical world have gained momentum. This also brings opportunities to contribute to new Crowd Management approaches, matching the objectives of both the environment and the individual users.

\section{References}

1. Liu, Z., Yang, D., Wen, D., Zhang, W.: Cyber-Physical-Social Systems for Command and Control. IEEE Intelligent Systems, Vol. 26, No. 4, 92-96. (2011)

2. De, S.; Zhou, Y.; Larizgoitia Abad, I.; Moessner, K.: CyberPhysicalSocial Frameworks for Urban Big Data Systems: A Survey. Appl. Sci. 2017, 7, 1017.

3. Candra Z.C.M., Truong H.L. and Dustdar, S.: Cyber-physical- social systems. 2016 IEEE World Congress on Services (SERVICES), San Francisco, California, USA, 2016; 56-63

4. Zeng, J., Yang, L. T., Lin, M., Ning, H.,and Ma, J.: A survey cyber-physical-social systems and their system-level design methodology. Future Generation Computer Systems, (2016).

5. Z. Su, Q. Qi, Q. Xu, S. Guo, and X. Wang.: Incentive scheme for cyber physical social systems based on user behaviours, IEEE Trans. Emerg. Topics Comput., to be published, doi: 10.1109/TETC.2017.2671843.

6. F.-Y.: Wang, The emergence of intelligent enterprises: From CPS to CPSS, IEEE Intell. Syst., vol. 25, no. 4, pp. 107110, 2010.

7. M Gharib, P Lollini, A Bondavalli.: Towards an Approach for Analyzing Trust in Cyber-Physical-Social Systems, IEEE System of Systems Engineering Conference (SoSE) 2017 
8. A. Sheth, P. Anantharam, and C. Henson.: Physical-Cyber-Social Computing: An Early 21st Century Approach, IEEE Intelligent Systems, vol. 28, no. 1, 2013, pp. 7982

9. A. Smirnov, T. Levashova, N. Shilov, K. Sandkuhl.: Ontology for Cyber-PhysicalSocial Systems Self-Organisation, Proceedings of the 16th Conference of Open Innovations Association FRUCT, Oulu, Finland, 27-31 October 2014, pp. 101-107.

10. Y. Zhu, Y. Tan, R. Li, and X. Luo .: Cyber-physical-social thinking modeling and computing for geological information service system. in Proceedings of the 4th International Conference on Identification, Information, and Knowledge in the Internet of Things (IIKI 15), Beijing, China, October 2015.

11. Z. Su, Q. Qi, Q. Xu, S. Guo, and X. Wang.: Incentive scheme for cyber physical social systems based on user behaviours. IEEE Trans. Emerg. Topics Comput., to be published, doi: 10.1109/TETC.2017.2671843.

12. S. G. Wang, A. Zhou, M. Yang, L. Sun, and C.-H. Hsu.: Service composition in cyber-physical-social systems. IEEE Trans. Emerg. Topics Comput., Feb. 2017, doi: 10.1109/TETC.2017.2675479

13. Huang, C.; Marshall, J.; Wang, D.; Dong, M.: Towards Reliable Social Sensing in Cyber-Physical-Social Systems. In Proceedings of the 2016 IEEE International Parallel and Distributed Processing Symposium Workshops (IPDPSW), Chicago, IL, USA, 2327 May 2016.

14. L. Aroyo, G. Antoniou, E. Hyvonen, A. Teije, H. Stuckenschmidt, L. Cabral, T. Tudorache, W. Hage, N. Stash, and Y. Wang.: Finding your way through the rijksmuseum with an adaptive mobile museum guide. The Semantic Web: Research and Applications, pp. 4659, 2010.

15. Y. Naudet, I. Lykourentzou, E. Tobias, A. Antoniou, J. Rompa, and G. Lepouras.: Gaming and cognitive profiles for recommendations in museums. in Proc. of the 8th Int. Workshop on Semantic and Social Media Adaptation and Personalisation (SMAP2013), 2013, pp. 6772.

16. I. Lykourentzou, X. Claude, Y. Naudet, E. Tobias, A. Antoniou, G. Lepouras, and C. Vasilakis.: Improving museum visitors Quality of Experience through intelligent recommendations: A visiting style-based approach, In Museums As Intelligent Environments (MasIE), Workshop co-located with the 9th International Conference on Intelligent Environments - IE13, Athens, Greece, July 16-19 2013

17. D. Surdilovic, G. Schreck, and U. Schmidt.: Development of collaborative robots (cobots) for flexible human-integrated assembly automation, In 41st Int. Symposium on Robotics, ISR, 2010.

18. T.M.Seban, J.M Salotti, B. Claverie, D. Bitonneau.: Classification of Cobotic Systems for Industrial Applications I towards a Framework for Joint Action, 2018.

19. K. J. Murakami.: CPSS (Cyber-physical-social System) Initiative-Beyond CPS (Cyber-Physical System) for a Better Future, Keynote Speech, The First JapanEgypt Conference on Electronics Communication and Computers JEC-ECC, Egypt, 2012 , .

20. D. Sousa Nunes, Pei Zhang, and J. Sa Silva.: A survey on human-in-the-loop applications towards an Internet of all. Communications Surveys Tutorials, IEEE, 17(2):944965, Secondquarter 2015.

21. A. Kouba and B. Andersson.: A vision of cyber-physical Internet, in Proc. Workshop RTN, Jul. 2009, pp. 16.

22. Y. Naudet, B.A Yilma and H.Panetto.: Personalisation in Cyber Physical and Social Systems: the Case of Recommendations in Cultural Heritage Spaces, on the 13th International Workshop on Semantic and Social Media Adaptation and Personalisation (SMAP 2018) 